\title{
Interferon- $\gamma$ therapy in two patients with progressive chronic pulmonary aspergillosis
}

\author{
P. Kelleher*\#, A. Goodsall", A. Mulgirigama+, H. Kunst" , D.C. Henderson*, \\ R. Wilson ${ }^{\#}$, A. Newman-Taylor ${ }^{+}$and M. Levin
}

\begin{abstract}
Infection by Aspergillus species causes a wide spectrum of pulmonary disease in humans.

In two patients with semi-invasive Aspergillus-induced lung disease, significantly reduced levels of interferon- $\gamma$ secretion by peripheral blood mononuclear cells were found after in vitro stimulation with the T-cell mitogen phytohaemagglutinin. Despite anti-fungal therapy, both patients exhibited progressive disease, and adjunctive interferon- $\gamma$ therapy was associated with significant clinical improvement.

The data suggest that impaired production of interferon- $\gamma$ can be seen in patients with chronic pulmonary aspergillosis. Adjunctive cytokine therapy with interferon- $\gamma$ may be useful in patients with progressive disease despite adequate anti-fungal therapy.
\end{abstract}

\section{KEYWORDS: Chronic pulmonary Aspergillus infection, interferon- $\gamma$ therapy}

I nfection with Aspergillus species can cause either allergic or invasive pulmonary disease in susceptible individuals. Invasive Aspergillus lung disease is associated with defects in phagocyte and T-cell function; acute invasive pulmonary infection can occur in bone marrow transplant recipients, whereas subacute forms of pulmonary aspergillosis are seen in patients with chronic granulomatous disease, HIV infection, diabetes mellitus and low-dose long-term corticosteroid therapy; however, many individuals do not show overt immune deficiency [1-3]. Experimental data from murine models of invasive aspergillosis and clinical observations of patients with invasive disease suggest that proinflammatory cytokines such as interferon (IFN)- $\gamma$, interleukin (IL)-12 and tumour necrosis factor (TNF)- $\alpha$ can play an important role in controlling this infection $[3,4]$. The present study describes the clinical features of two patients with chronic pulmonary aspergillosis who showed impaired IFN- $\gamma$-mediated immune responses, and in whom adjunctive therapy with this cytokine was associated with significant clinical improvement.

\section{PATIENTS AND METHODS}

Patients with confirmed or suspected atypical mycobacterial chest disease are screened for the presence of type 1 cytokine (IL-12 and IFN- $\gamma$ ) deficiency. Investigations for IL-12 or IFN- $\gamma$ deficiency are carried out as follows: peripheral blood mononuclear cells (PBMCs) are isolated following density centrifugation and suspended at a concentration of $1 \times 10^{6}$ cells $\cdot \mathrm{mL}^{-1}$ in culture medium. IFN- $\gamma$, IL-12p40 and IL-12p70 levels are measured using an ELISA (BD Biosciences, Oxford, UK) in accordance with the manufacturer's instructions after stimulation with the Tcell mitogen phytohaemagglutinin (PHA) [5]. The integrity of the IL-12 receptor pathway is determined by measuring the concentration of IFN- $\gamma$ after the addition of exogeneous IL-12 to PBMC cultures $[6,7]$. The concentration of TNF- $\alpha$ is measured by ELISA after stimulation with lipopolysaccharide alone or in combination with IFN $-\gamma$ in order to assess the function of the IFN- $\gamma$ receptor and signal transduction pathway $[6,7]$. Patients are also screened for the presence of neutralising anti-IFN- $\gamma$ autoantibodies [8]. The concentration of IFN- $\gamma$ in PBMCs isolated from a healthy individual after stimulation with PHA and IL-12 in cultures containing $2.5-10 \%$ patient serum was measured by ELISA. In this in vitro system, increasing concentrations of patient serum containing neutralising anti-IFN- $\gamma$ autoantibodies are associated with a significant fall in
AFFILIATIONS

*Dept of Immunology, Imperial College, Chelsea \& Westminster Hospital,

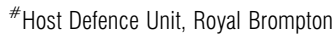
Hospital,

"Dept of Academic Paediatrics, Wright Fleming Building, Imperial College, St Mary's Hospital, and +Dept of Respiratory Medicine and the National Heart and Lung Institute, Imperial College, Royal Brompton Hospital, London, UK.

CORRESPONDENCE

P. Kelleher

Dept of Immunology

Imperial College

Chelsea \& Westminster Hospital

369 Fulham Road

London SW10 9NH

UK

Fax: 4420874667559

E-mail: p.kelleher@imperial.ac.uk

Received:

February 242005

Accepted after revision:

October 132005

SUPPORT STATEMENT

The study on type 1 cytokine deficiency was supported by a Wellcome Trust (London, UK) grant to M. Levin. The authors declare that they have no conflict of interest. 
the ability of PBMCs isolated from a healthy individual to secrete this cytokine. In all cases in whom a defect in IFN- $\gamma$ production is noted after PHA-induced T-cell stimulation, the cytokine studies are repeated to confirm the results obtained. Two patients with suspected atypical mycobacterial disease were found to have chronic invasive cavitating pulmonary aspergillosis, and the case reports of these individuals are outlined below.

\section{CASE REPORTS}

\section{Case 1}

A 37-yr-old female was admitted with a 3-month history of productive cough, fever, haemoptysis and malaise. In the previous year, she had experienced a cavitating pneumonia, which failed to respond to conventional antibiotics. Bronchoscopy demonstrated purulent secretions in the right upper lobe and bronchoalveolar lavage fluid (BALF) culture yielded Pseudomonas aeruginosa and Aspergillus niger with fungal hyphae. Acid-fast bacilli were not grown. She was treated for both Pseudomonas and A. niger pneumonia and showed slow clinical improvement, with persistent chest radiographic abnormalities. She had a previous history of bilateral recurrent pneumothoraces between the ages of 29 and $31 \mathrm{yrs}$, which were treated by bilateral pleurectomy. A computed tomography (CT) scan at this time revealed no parenchymal lung disease and a follow-up chest CT scan at the age of 33 yrs showed minor upper lobe fibrosis. Screening tests for cystic fibrosis gave negative results.

On the present admission, sputum and BALF culture yielded $A$. fumigatus and a chest CT scan showed bilateral upper lobe cavitation with adjacent pleural thickening and parenchymal destruction. One left upper lobe cavity contained a mycetoma and left lower lobe consolidation was seen with ill-defined margins. Transbronchial lung biopsy results were consistent with a focal organising pneumonia; however, no fungal hyphae were detected. The patient gave a positive Aspergillus precipitins (immunoglobulin $(\mathrm{Ig}) \mathrm{G}$ ) result, and the results of specific IgE testing and a skin-prick test for $A$. fumigatus were both negative. Her C-reactive protein levels were raised. The clinical and radiological presentation was felt to be consistent with early chronic pulmonary aspergillosis. The patient improved on a combination of broad-spectrum antibiotics and itraconazole and was discharged home on itraconazole $200 \mathrm{mg}$ b.i.d.

Immunological investigations revealed positive rheumatoid factor test results of 1:5,012 (normal $<1: 40$ ) and a reduced CD4+ T-cell count of $3.4 \times 10^{8}$ cells $\cdot \mathrm{L}^{-1}$ (reference interval $4 \times 10^{8}-1.2 \times 10^{9}$ cells $\cdot \mathrm{L}^{-1}$ ). Serum immunoglobulins, tetanus, Haemophilus influenzae $\mathrm{b}$ and pneumococcal antibody levels were normal. T-cell proliferation in response to mitogens (PHA and concanavalin A) and antigens (Siebert purified protein derivative of tuberculin (PPD), candida, tetanus and measles) was normal. Neutrophil function tests revealed a normal respiratory oxidative burst, phagocytosis of Staphylococcus aureus and expression of CD18 and CD11b adhesion markers. A HIV antibody test was negative. Analysis of IFN- $\gamma$ production following PHA stimulation of PBMC cultures revealed a marked defect in the secretion of this cytokine compared with healthy controls (table 1). There was no evidence of either a complete or partial IFN- $\gamma$ receptor

\begin{tabular}{|c|c|c|c|}
\hline \multirow[t]{3}{*}{ TABLE 1} & \multicolumn{3}{|c|}{$\begin{array}{l}\text { In vitro production of interferon (IFN)- } \gamma \text { by } \\
\text { affected patients and healthy controls following } \\
\text { phytohaemagglutinin (PHA) and interleukin (IL)- } \\
12 \text { stimulation }\end{array}$} \\
\hline & \multicolumn{3}{|c|}{$\mathrm{IFN}-\gamma \mathrm{pg} \cdot \mathrm{mL}^{-1}$} \\
\hline & Controls ${ }^{\#}$ & Case 1 & Case 2 \\
\hline PHA & $11759 \pm 6122(3613-19989)$ & 1000 & 2239 \\
\hline PHA+IL-12 & $41201 \pm 19957(9307-65875)$ & 15500 & 14252 \\
\hline
\end{tabular}

\begin{tabular}{|c|c|c|c|}
\hline \multirow[t]{3}{*}{ TABLE 2} & \multicolumn{3}{|c|}{$\begin{array}{l}\text { In vitro production of tumour necrosis factor } \\
\text { (TNF)- } \alpha \text { by affected patients and healthy controls } \\
\text { following lipopolysaccharide (LPS) and interferon } \\
\text { (IFN)- } \gamma \text { stimulation }\end{array}$} \\
\hline & \multicolumn{3}{|c|}{ TNF- $\alpha \mathrm{pg} \cdot \mathrm{mL}^{-1}$} \\
\hline & Controls ${ }^{\#}$ & Case 1 & Case 2 \\
\hline LPS & $1097 \pm 596(494-1942)$ & 2087 & 2629 \\
\hline LPS + IFN- $\gamma$ & $3837 \pm 1767(303-7371)$ & 10166 & 8199 \\
\hline
\end{tabular}

deficiency or impaired IL-12 receptor function (tables 1 and 2 ). The concentration of IL-12p40 and IL-12p70 was similar to that in healthy controls and this patient's serum did not contain neutralising anti-IFN- $\gamma$ autoantibodies.

The patient experienced a further relapse of A. fumigatus semiinvasive aspergillosis 7 months after this presentation, which responded to intravenous caspofungin therapy. She was also commenced on subcutaneous IFN- $\gamma\left(60 \mu \mathrm{g} \cdot \mathrm{m}^{-2}\right)$ three times a week. Currently, she remains symptomatically stable on voriconazole $200 \mathrm{mg}$ b.i.d. and subcutaneous IFN- $\gamma$ and has not required hospital admission to treat her condition for 2 yrs.

\section{Case 2}

A 56-yr-old female was referred with a 6-month history of productive cough, dyspnoea, occasional haemoptysis, lethargy and weight loss of $3.5 \mathrm{~kg}$. She had been treated with several courses of broad-spectrum antibiotics with little effect. She had a past history of recurrent left-sided pneumothoraces, which were treated by pleurodesis at the age of $22 \mathrm{yrs}$. Ten years previously, she had been diagnosed with Mycobacterium kansasii chest infection and had received antituberculosis therapy for this condition for 3 yrs. Her identical twin also had a history of recurrent pneumothoraces and atypical mycobacterial chest infection. She showed an elevated C-reactive protein concentration, which had not fallen with antibacterial therapy. A chest CT scan showed severe bilateral apical fibrocavitatory disease, adjacent pleural thickening and a cavitating nodule in the right lower lobe. Lung function tests showed a restrictive defect and reduced gas transfer. Repeated sputum and BALF cultures were negative for mycobacteria; 
however, A. fumigatus was cultured from her sputum and she gave positive Aspergillus precipitins (IgG) results. A sweat test provided normal results.

Immunological investigations revealed positive rheumatoid factor results of 1:640; other autoimmune serological results were negative. Serum Igs showed a polyclonal rise in IgM to $7.0 \mathrm{~g} \cdot \mathrm{L}^{-1}$ (reference interval $\left.0.4-2.0 \mathrm{~g} \cdot \mathrm{L}^{-1}\right)$, with a marked reduction in serum $\operatorname{IgA}\left(<0.1 \mathrm{~g} \cdot \mathrm{L}^{-1}\right)$ and $\operatorname{IgE}\left(<0.3 \mathrm{IU} \cdot \mathrm{mL}^{-1}\right)$ levels. She showed a slightly reduced CD4+ T-cell count of $3.4 \times 10^{8}$ cells $\cdot \mathrm{L}^{-1}$ (reference interval $4.5 \times 10^{8}-1.66 \times 10^{9}$ cells $\cdot \mathrm{L}^{-1}$ ). Tetanus, $H$. influenzae $\mathrm{b}$ and pneumococcal antibody levels were normal. T-cell proliferation in response to mitogens (PHA and concanavalin A) and antigens (PPD, candida, tetanus and measles) was normal. Neutrophil function tests revealed a normal respiratory oxidative burst, phagocytosis of $S$. aureus and expression of CD18 and CD11b adhesion markers. There was a significant reduction in IFN- $\gamma$ secretion following PHA stimulation in PBMC cultures (table 1). The concentration of IL-12p40 and IL-12p70 was similar to that in healthy controls. There was no evidence of either a complete or partial IFN- $\gamma$ receptor deficiency or impaired IL-12 receptor function (tables 1 and 2). This patient's serum contained no neutralising anti-IFN- $\gamma$ autoantibodies.

The patient was started on standard antituberculosis therapy and itraconazole $100 \mathrm{mg}$ b.i.d. pending the results of her tuberculosis cultures. She remained unwell with subtherapeutic itraconazole levels, due in part to accelerated catabolism by rifampicin. Her tuberculosis medications were stopped when the results of BAL cultures revealed no mycobacterial organisms. The dose of itraconazole was increased to $400 \mathrm{mg}$ b.i.d. in order to achieve a satisfactory minimal inhibitory concentration. After 1 month on this treatment, she developed left ventricular failure and was switched to voriconazole. She exhibited a poor clinical response to this agent and showed deteriorating pulmonary function test results and new pulmonary cavitating lesions on chest CT scan. At this stage, subcutaneous IFN- $\gamma 50 \mu \mathrm{g} \cdot \mathrm{m}^{-2}$ three times a week was added to her antifungal treatment. She showed a significant improvement in chest symptoms, a reduction in inflammatory marker levels and an increase in lung volumes, transfer factor and exercise capacity. The IFN- $\gamma$ therapy was discontinued after 8 months' therapy because of the improvement in her clinical condition, which made the side-effects of IFN- $\gamma$ less tolerable. She continued on voriconazole $200 \mathrm{mg}$ daily for a further 4 months before stopping this treatment. She remained well for 15 months until she relapsed with cavitating pulmonary aspergillosis and was recommenced on voriconazole. As before, she showed a limited clinical response to this agent and her symptoms and inflammatory markers only improved after the introduction of IFN- $\gamma$.

\section{DISCUSSION}

In two patients with Aspergillus-induced lung disease, significantly reduced levels of IFN- $\gamma$ were found in PBMC cultures stimulated in vitro with the T-cell mitogen PHA (table 1). Both patients had progressive disease despite antifungal therapy, and adjunctive IFN- $\gamma$ therapy was associated with significant clinical improvement.
One limitation of the present study is the failure to isolate fungal hyphae from tissue biopsy specimens or culture Aspergillus species from a sterile site; however; transbronchial biopsy or percutaneous aspirates often have a low diagnostic yield [9]. The present patients meet the criteria outlined by DENNING et al. [10] for enrolment into prospective studies of chronic pulmonary aspergillosis. Genetic defects in the expression of the IFN- $\gamma$ receptor, IL-12 $\beta 1$ receptor or IL-12/ IL-23p40, resulting in impaired T-cell production of IFN- $\gamma$, are well established causes of atypical mycobacterial disease [11]. As there is a strong correlation between IFN- $\gamma$ receptor genotype and cellular responsiveness to this cytokine, the present data exclude the presence of a complete or partial IFN- $\gamma$ receptor 1 deficiency in the two patients studied [12]. The two patients are unlikely to have defective IFN- $\alpha / \beta$ immune responses as the only known mutation affecting this signalling pathway also impairs cell responsiveness to IFN- $\gamma$ [13]. IFN- $\gamma$ levels were upregulated after stimulation with PHA and IL-12, which may make it highly unlikely that the present patients have a defect in the expression of IL-12 $\beta 1$ receptor. Neither patient had reduced or absent IL-12/IL23p40 or IL-12p70 concentrations. Neutralising anti-IFN- $\gamma$ autoantibodies, which may inhibit the secretion of IFN- $\gamma$ from T-cells, were not found in the present patients, although the possibility that both individuals may have a defect in IFN- $\gamma$ cellular secretion pathway cannot be excluded [8, 14, 15]. Further studies are required to establish whether these two patients are deficient in other proteins, such as IL-18 or IL-27, which are also associated with type- 1 T-helper cell cytokine responses. The role of cytokines, such as IL-10, transforming growth factor- $\beta$, IL-4 and IL-13, which can either inhibit the production or antagonise the actions of IFN- $\gamma$, should also be evaluated in these two patients. The prevalence of IFN- $\gamma$ deficiency in patients with Aspergillus-related lung disease and the role of adjunctive cytokine therapy, such as IFN- $\gamma[10,16,17]$, in patients with impaired IFN- $\gamma$ immunity and progressive disease despite appropriate antifungal therapy needs to be clarified.

The results of this preliminary study are consistent with other findings suggesting that IFN- $\gamma$-mediated immune responses play an important role in the control of fungal infections in humans. Patients with the hyper-IgE syndrome, who are at increased risk of invasive Aspergillus infection, show a selective defect in the secretion of IFN- $\gamma$ [18]. The recovery of bone marrow transplantation patients with invasive Aspergillus infection is associated with increased IFN- $\gamma / \mathrm{IL}-10$ ratios in cell culture supernatants compared with those seen in patients who died because of this infection [19]. The mechanisms leading to impairment of IFN- $\gamma$ production have not been identified in these patients.

In conclusion, it has been shown that two patients with chronic invasive pulmonary aspergillosis exhibit significant defects in interferon- $\gamma$-mediated immune responses, and that treatment of this condition with this cytokine results in significant clinical improvement. Recognition of impaired interferon- $\gamma$ immunity may identify patients who require adjunctive cytokine therapy to control this infection.

\section{ACKNOWLEDGEMENTS}

The authors would like to thank D. Hansell (Dept of Radiology, Royal Brompton \& Harefield NHS Trust, London, 
UK) for helpful comments concerning the computed tomography scans for case 2, A. Hall and N. Saunders (both Dept of Immunology, Chelsea \& Westminster Hospital, London, UK) for help with the neutrophil function and T-cell proliferation assays, and F. Gotch (Dept of Immunology, Chelsea \& Westminster Hospital, Imperial College, London, UK) and D. Denning (School of Medicine, Wythenshawe Hospital, University of Manchester, Manchester, UK) for helpful comments on this article.

\section{REFERENCES}

1 Soubani AO, Chandrasekar PH. The clinical spectrum of pulmonary aspergillosis. Chest 1999; 121: 1989-1999.

2 Denning DW. Invasive aspergillosis. Clin Infect Dis 1998; 26: 781-805.

3 Kontoyiannis DP, Bodey GP. Invasive aspergillosis in 2002: an update. Eur J Clin Microbiol Infect Dis 2002; 21: 161-172.

4 Romani L. Immunity to fungal infections. Nat Rev Immunol 2004; 4: 11-24.

5 Weir RE, Morgan AR, Britton WJ, Butlin CR, Dockrell HM. Development of a whole blood assay to measure $\mathrm{T}$ cell responses to leprosy: a new tool for immuno-epidemiological field tools of leprosy immunity. J Immunol Methods 1994; 176: 93-101.

6 Levin M, Newport MJ, D'Souza S, et al. Familial disseminated atypical mycobacterial infection in childhood: a human mycobacterial susceptibility gene. Lancet 1995; 345 : 79-83.

7 Jouanguy E, Lamhamedi-Cherradi S, Altare F, et al. Partial interferon- $\gamma$ receptor 1 deficiency in a child with tuberculoid bacillus Calmette-Guérin infection and a sibling with clinical tuberculosis. J Clin Invest 1997; 100: 2658-2664.

8 Doffinger R, Helbert MR, Barcenas-Morales G, et al. Autoantibodies to interferon- $\gamma$ in a patient with selective susceptibility to mycobacterial infection and organ-specific autoimmunity. Clin Infect Dis 2004; 38: e10-e14.
9 Saraceno JL, Phelps DT, Ferro TJ, Futerfas R, Schwartz DB. Chronic necrotizing pulmonary aspergillosis: approach to management. Chest 1997; 112: 541-548.

10 Denning DW, Riniotis K, Dobrashian R, Sambatakou H. Chronic cavitary and fibrosing pulmonary and pleural aspergillosis: case series, proposed nomenclature change, and review. Clin Infect Dis 2003; 37: Suppl. 3, S265-S280.

11 Casanova J-L, Abel L. The human model: a genetic dissection of immunity to infection in natural conditions. Nat Rev Immunol 2004; 4: 55-65.

12 Dorman S, Picard C, Lammas D, et al. Clinical features of dominant and recessive interferon $\gamma$ receptor 1 deficiencies. Lancet 2004; 364: 2113-2121.

13 Dupuis S, Jouanguy E, Al-Hajjar S, et al. Impaired response to interferon $\alpha / \beta$ and lethal viral disease in human STAT1 deficiency. Nat Genet 2003; 33: 388-391.

14 Hofflich C, Sabat R, Rosseau A, et al. Naturally occurring anti-IFN- $\gamma$ autoantibody and severe infections with Mycobacterium chelonae and Burkholderia cocovenenans. Blood 2004; 103: 673-675.

15 Safdar A, Armstrong D. A novel defect in interferon- $\gamma$ secretion in patients with refractory nontuberculous pulmonary mycobacteriosis. Ann Intern Med 2003; 138: 521.

16 Bernhisel-Broadbent J, Camargo EE, Jaffe HS, Lederman HM. Recombinant human interferon- $\gamma$ as adjunct therapy for Aspergillus infection in a patient with chronic granulomatous disease. J Infect Dis 1991; 163: 908-911.

17 Rex JH, Bennett JE, Gallin JI, Malech HL, DeCarlos ES, Melnick DA. In vivo interferon- $\gamma$ therapy augments the in vitro ability of chronic granulomatous disease neutrophils to damage Aspergillus hyphae. J Infect Dis 1991; 163: 849-852.

18 Ito R, Mori M, Katakura S, et al. Selective insufficiency of IFN- $\gamma$ secretion in patients with the hyper-IgE syndrome. Allergy 2003; 58: 329-336.

19 Hebart H, Bollinger C, Fisch P, et al. Analysis of T-cell responses to Aspergillus fumigatus antigens in healthy individuals and patients with hematologic malignancies. Blood 2002; 100: 4521-4528. 